\title{
Remote-sensing Fusion by Multiscale Block-based Compressed Sensing
}

\author{
Yang Senlin ${ }^{1, ~ a, ~ C h o n g ~} \mathrm{Xin}^{2, \mathrm{~b}}$ \\ ${ }^{1}$ School of Mechatronic and Material Engineering, Xi'an University, Xi'an, Shaanxi 710065, P. R. \\ China \\ ${ }^{2}$ Department of Power, Emerson Network Power Ltd., Xi'an 710075, Shaanxi, P. R. China

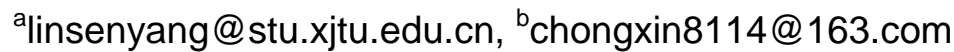

Keywords: Compressed sensing, Remote sensing, Image fusion, Iterative thresholding projection.

\begin{abstract}
A new compressive fusion algorithm based on non-uniform sampling is proposed. Although conventional block-based compressed sensing (BCS) represents a low computational cost, it suffers from low reconstruction quality since it is not well accounting for global image features. Employing the structured random matrix, multiscale non-uniform BCS (MNBCS) is implemented with decomposition level-dependent block-sizes and subrates. The proposed methodology improves the reconstruction quality without impacting the computational complexity. Experimental results show that the iterative soft-thresholding projection (ISTP) reconstruction with MNBCS achieves a higher reconstruction quality and a lower computational cost than. At very low sampling rates, MNBCS outperforms traditional wavelet-based fusion techniques.
\end{abstract}

\section{Introduction}

Image fusion is widely applied in remote sensing, medical imaging, military surveillance, homeland security, environmental protection, traffic monitoring, and disaster forecasting. The popular fusion techniques are based on multiresolution decomposition schemes such as wavelet transform, curvelet transform, and contourlet transform [1]. However, these fusion methods require all information of the input images, which is inconvenient for handling large images. Hence, the question arises whether fusion can be taken based upon partial information retrieved from the input images without affecting the final art. This is exactly the functionality that compressed sensing (CS) theory [2] is offering since it allows for reconstructing the fused image from a limited set of samples. Nonetheless, state-of-the-art solutions typically adhere to the global compressive sampling approach, which results in huge memory requirements and an expensive computational cost. The block-based compressed sensing (BCS) [2] enables fast computation and imposes small memory requirements, but it suffers from a low reconstruction quality since the BCS defects global features. Hence in this paper, we propose a novel compressive fusion method based on BCS and multiscale transforms for retaining global features to alleviate the drawbacks of BCS solutions.

The remainder of the paper is organized as follows. First, in Section 2, we provide a brief description of non-uniform BCS in the multiscale transform domain. Then, in Section 3, we describe the fusion strategy for multiscale compressive sampling. In Section 4, we provide the reconstruct algorithm of fused image, and, in Section 5, we present the experimental results. Finally, conclusions are drawn in Section 6.

\section{Multiscale Non-uniform BCS Sampling}

Consider a vectorized image $\boldsymbol{x} \in \mathbb{R}^{N \times 1}$ and suppose that an orthogonal basis $\boldsymbol{\psi} \in \mathbb{R}^{N \times N}$ provides a $\kappa$-sparse representation of $\boldsymbol{x}$. In terms of matrix notation, we have $\boldsymbol{x}=\boldsymbol{\psi} \boldsymbol{s}$, in which $\boldsymbol{s} \in \mathbb{R}^{N \times 1}$ can be well-approximated using only $\kappa<<N$ non-zero entries. The CS theory states that such a signal $\boldsymbol{x}$ can be reconstructed by taking only $M=O(\kappa \log N)$ linear, non-adaptive projections with $\boldsymbol{y}=\boldsymbol{\Phi} \boldsymbol{x}$, in which $\boldsymbol{y} \in \mathbb{R}^{M \times 1}$ represents the compressive sampled signal and $\Phi \in \mathbb{R}^{M \times N}(M<<N)$ is the 
measurement matrix. Obviously, since the projection is an ill-posed inverse problem, an infinite number of solutions exist. In order to reconstruct the $\kappa$-sparse signal $\boldsymbol{x}$, the measurement matrix and sparse basis should satisfy the restricted isometric property (RIP) condition [2].

To increase computational efficiency, the large image can be divided into $B \times B$ blocks and then sampled by an identical sampling operator $\Phi_{B} \in \mathbb{R}^{M_{B} \times B^{2}}\left(M_{B}<<B^{2}\right)$. Let $\boldsymbol{x}_{i} \in \mathbb{R}^{B^{2} \times 1}$ represent the vectorized signal of block $i$, then the block-based compressed sensing (BCS) measurement is $\boldsymbol{y}_{i}=\boldsymbol{\Phi}_{B} \boldsymbol{x}_{i}$, where $\boldsymbol{y}_{i} \in \mathbb{R}^{M_{B} \times 1}$. Note that BCS is memory efficient since we only need to store an $M_{B} \times B^{2}$ sampling matrix $\Phi_{B}$.As a result, BCS-based techniques can enable fast reconstruction, but suffer from reduced reconstruction quality due to their reliance on block-based observations.

Therefore, it is advisable to deploy BCS fusion and multiresolution-based methods since the global features can be accounted at different scale in transform domain. Here, the multiscale transform is preferred to choose the 2D discrete wavelet transform (DWT) operator $\Psi \in \mathbb{R}^{N \times N}$ due to its fast implementation. Assume that $s \in \mathbb{R}^{N \times 1}$ are the vectorized coefficients of DWT decomposition of the image $\boldsymbol{x} \in \mathbb{R}^{N \times 1}$ with $L$ levels, i.e., $\boldsymbol{s}=\boldsymbol{\Psi} \boldsymbol{x}$. For the 2D representation of DWT decomposition $\boldsymbol{s}$, suppose that the DWT coefficients in subband $j \subset\{H, V, D\}$ at level $l$ are divided into $B_{l} \times B_{l}$ blocks and then sampled with a level-dependent sampling operator $\Phi_{l} \in \mathbb{R}^{M_{l} \times B_{l}^{2}}\left(M_{l}<<B_{l}^{2}\right)$. For convenience, let subscript $v$ represent the index set $\{l, j, i\}$, and $\boldsymbol{s}_{v} \in \mathbb{R}^{B_{l}^{2} \times 1}$ denote the vectorized coefficients of block $i$ in subband $j$ at level $l$, we get

$$
\boldsymbol{y}_{v}=\boldsymbol{\Phi}_{l} \boldsymbol{s}_{v}
$$

where $\boldsymbol{y}_{v} \in \mathbb{R}^{M_{l} \times 1}$ is the multiscale BCS sampling at index $v=\{l, j, i\}$ for image $\boldsymbol{x}$, and $M_{l}$ is the number of samples. The ratio between $M_{l}$ and $B_{l}^{2}$ is the sampling ratio, i.e., the subrate $c_{l}$. For the measurement matrix, the structurally random matrix (SRM) [3] is employed because it can provide optimal sampling number and better recovery for block processing.

Since coefficients from various decomposition levels contribute differently to the recovery quality, recovery may be enhanced by non-uniform subrates for different subbands. As such, a larger subrate can be attributed to the subband contributing more significantly to the recovery quality, and vice versa. For convenience, let the level $l=0$ correspond to the lowest-frequency subband of DWT decomposition. As a result, the overall target subrate $c$ is

$$
c=\frac{1}{N} \sum_{l=0}^{L}\left(c_{l} \sum_{j=1}^{J_{l}} n_{l, j}\right),
$$

where $L$ represents the number of scales, $N$ is the number of total pixels, $J_{l}$ is the number of subbands at level $l$, and $n_{l, j}$ is the number of coefficients in subband $j$ at level $l$. Since such kind of compressive sampling results in non-uniform sampling for different levels, we termed this kind of compressive measurements as multiscale based non-uniform BCS samples (MNBCS) in the paper.

\section{Compressive Fusion Rules}

Traditionally, the multiscale decompositions of the input images are fused by linear weighting and maximum absolute value, which badly preserve the edge characteristics since they operate on single coefficients. For preserving the edge characteristics, the local characteristic fusion is introduced. On one hand, for MNBCS of the sparse approximation coefficients, the criterion of maximum local energy is introduced for fusion. On the other hand, the maximum local variance rule is employed for MNBCS sampling of the detail coefficients. Therefore, the fusion is determined by the neighboring MNBCS samples in a particular subband, which can well preserve the edge characteristics. 


\section{Reconstruction after fusion}

The final fused image can be reconstructed after the MNBCS samplings have been fused. For CS reconstruction, a number of methodologies have been proposed such as linear programming, gradient projection sparse reconstruction (GPSR), iterative greedy methods, and iterative thresholding methods [3]. However, these methods are always of high computational complexity or low recovery quality[4]. The iterative soft-thresholding projection (ISTP) [5] is capable of fast reconstruction with good quality. Therefore, we employ ISTP in our solution. We define the iterative soft-thresholding function as

$$
S\left(\Psi, \Theta, \boldsymbol{\theta}^{(n)}\right)=\Psi \Theta^{-1} \tilde{\boldsymbol{w}}^{(n)},
$$

where $\boldsymbol{\theta}^{(n)}$ is the $n$-th iteration of the multiscale coefficients, $\Theta \in \mathbb{R}^{N \times N}$ is a directional transform operator for thresholding filtering. In equation (6), $\tilde{\boldsymbol{w}}^{(n)}$ denotes the thresholding estimation of the directional coefficients $\boldsymbol{w}^{(n)}=\Theta \Psi^{-1} \tilde{\boldsymbol{\theta}}^{(n)}$, in which $\tilde{\boldsymbol{\theta}}^{(n)}$ is the block-based iterative projection of $\boldsymbol{\theta}^{(n)}$ on a convex set. For iteration $\boldsymbol{\theta}_{v}^{(n)}$ at an arbitrary index $v$, the iterative projection on a convex set is defined by $\tilde{\boldsymbol{\theta}}_{v}^{(n)}=\boldsymbol{\theta}_{v}^{(n)}+\boldsymbol{\Phi}_{l}^{T}\left(\boldsymbol{Y}_{v}-\boldsymbol{\Phi}_{l} \boldsymbol{\theta}_{v}^{(n)}\right)$.

\section{Results}

The recovery performance of ISTP method with MNBCS is firstly examined. For the PAN and MS images shown in Figure 1, both having a size of $1024 \times 1024$ pixels, are decomposed by a 3-level DWT and sampled at a subrate of 0.25 . The normalized iteration error of the ISTP, and peak signal-to-noise ratios (PSNRs) at various subrates for MNBCS and BCS is given in Figure 2, which implies that the computational efficiency and recovery quality of ISTP with MNBCS outperforms than that with BCS.
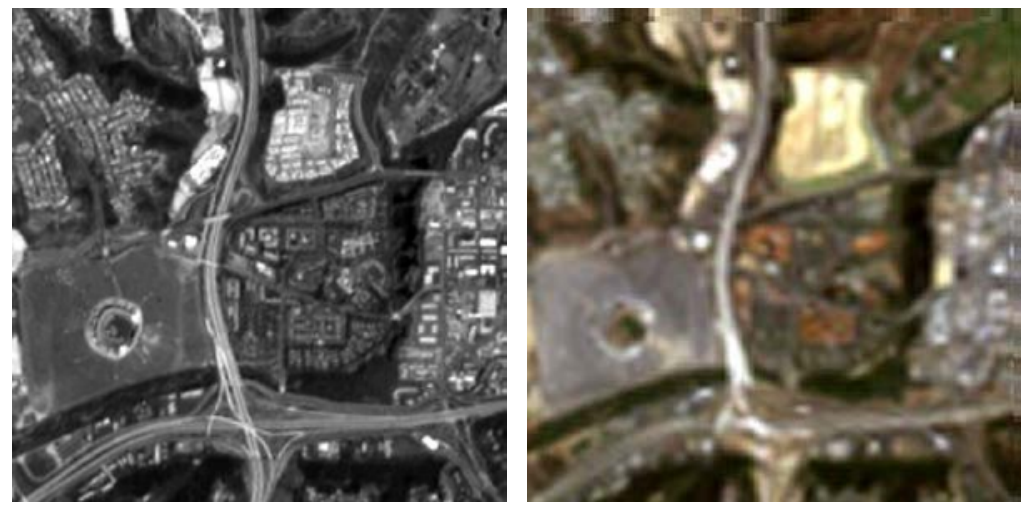

Figure. 1. The original PAN (left) and MS (right) images
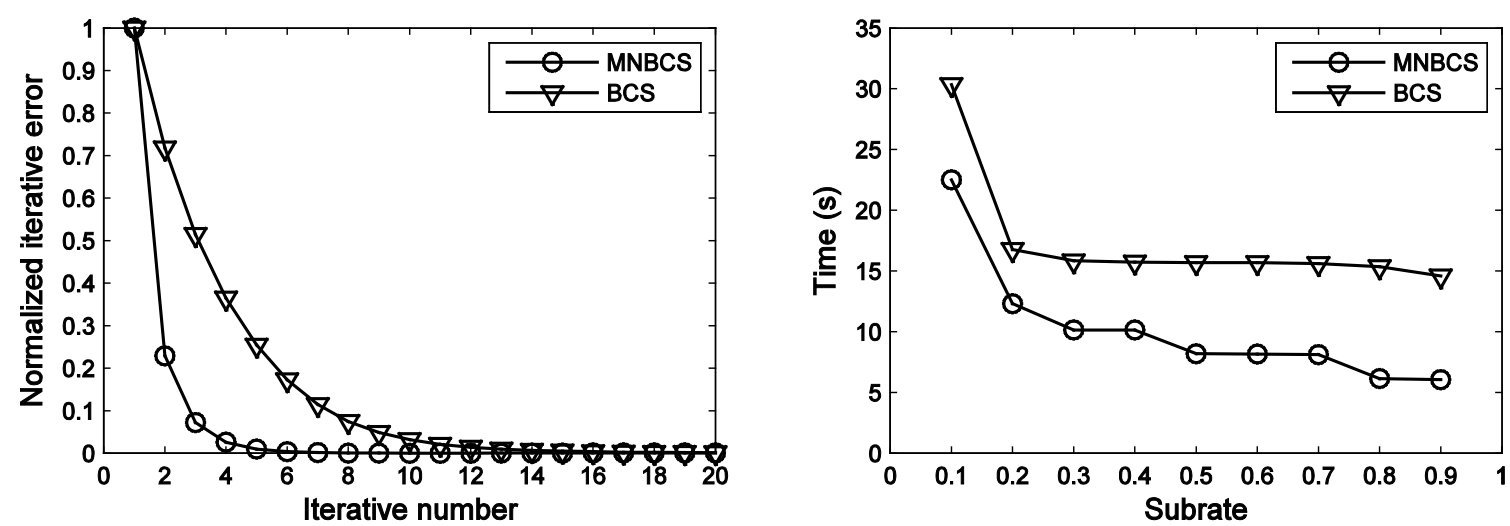

Figure. 2. The iterative errors (left) and PSNRs of the recovery image (right). 

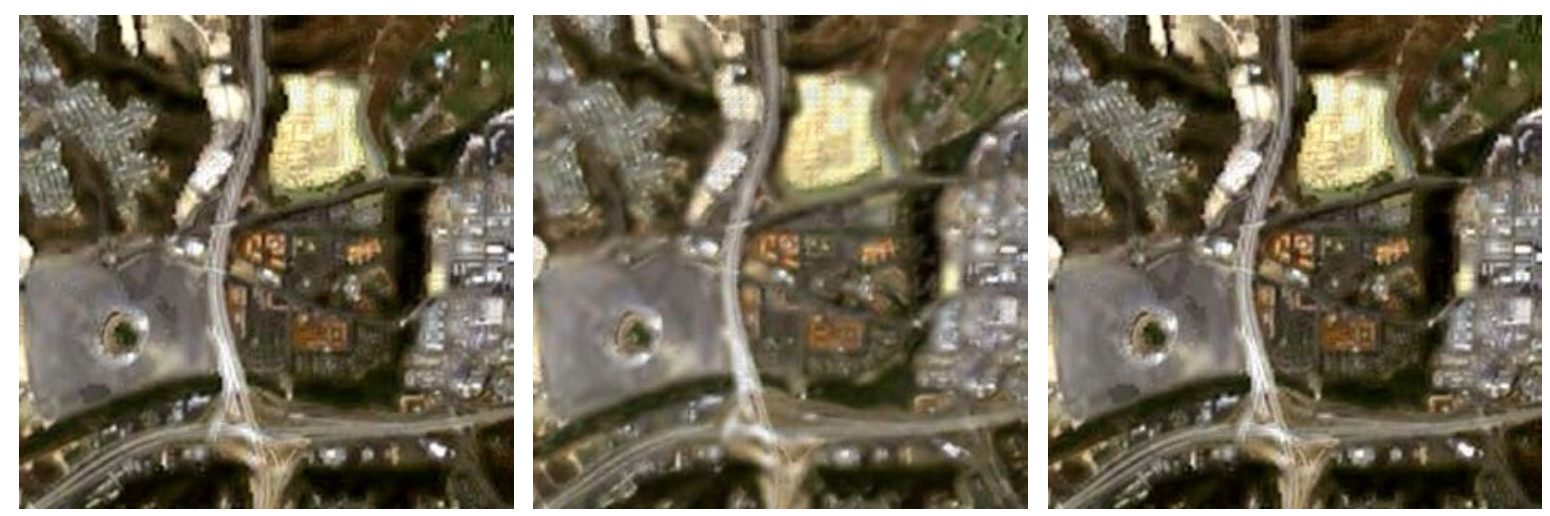

Figure. 3. Fusion results of MNBCS (left), BCS (middle), and DWT (right).

For field remote-sensing images, Figure 3 show fused results the MNBCS (left), BCS (middle) and conventional DWT (right), respectively. Additionally, the quantitative analysis of these fused images is given in Table 1. The utilized metrics include the mean value (MV), the standard deviation (STD), the information entropy (IE) and the average gradient (AG). Clearly, compressive fusion with local features produces more convincing results in STD, IE and AG than that with BCS, which shows smaller difference in values of quantitative analysis with that of traditional DWT fusion.

Table 1. Quantitative analysis for different fusion images

\begin{tabular}{lrrrl}
\hline Method & \multicolumn{1}{c}{ MV } & STD & AG & IE \\
\hline MNBCS & 88.78 & 55.95 & 3.94 & 2.30 \\
BCS & 101.49 & 50.19 & 3.46 & 2.29 \\
DWT & 88.78 & 56.14 & 4.32 & 2.30 \\
\hline
\end{tabular}

\section{Conclusion}

In this paper, we presented a compressive fusion strategy with MNBCS for remote-sensing imagery, which accounts for global image features. Numerical results illustrate that iterative soft-thresholding projection in combination with MNBCS achieves a better reconstruction quality at a lower computational cost than that based on BCS approach. The results of field test demonstrate that the proposed method produces comparable fusion results with traditional DWT-based fusion while requiring a smaller number of samples.

\section{Acknowledgements}

The work was partially supported by the Natural Science Fund of China (grant 61401356), the Natural Science Foundation Fund of Shaanxi Province grant 2013k07-47, and Xi'an Science and Technology Planning Projects (grant CXY1531WL06, CXY1531WL36) .

\section{References}

[1] X. Yang , L. Jiao, Acta Automatica Sinica, vol.34(2008), p.274-281.

[2] J. E. Fowler, M. Sungkwang and E.W Tramel, Foundations and Trends in Signal Processing, vol. 4(2010), p. 297-416.

[3] T.T. Do, T.D. Trans, L. Gan, Proc. ICASSP, Mar.31-Apr.4, p.3369 - 3372, 2008.

[4] J.E. Fowler, S. Mun and E.W. Tramel, Proc. 19th EUSIPCO, Aug.29-Sep.2, p.564-568, 2011.

[5] J. Bobin, J. Starck and R. Ottensamer, IEEE J. Sel. Topics Signal Process., vol.2(2008),p. 718-726. 